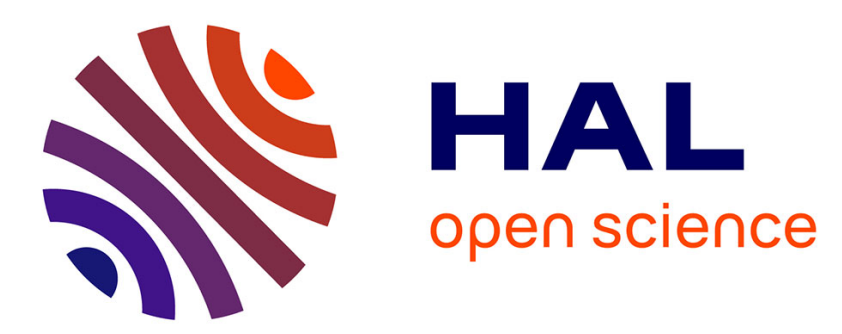

\title{
Three-Dimensional Reconstructions in Microscopy From Two-Dimensional Interferograms Using Sparsity-Inducing Regularization
}

\author{
L. Bonnefois, L. Mugnier, L. Houillot, L. Druart, L. Blanco
}

\section{- To cite this version:}

L. Bonnefois, L. Mugnier, L. Houillot, L. Druart, L. Blanco. Three-Dimensional Reconstructions in Microscopy From Two-Dimensional Interferograms Using Sparsity-Inducing Regularization. Imaging and Applied Optics, Optics and Photonics Congress, Jul 2016, HEIDELBERG, Germany. hal-01384152

\author{
HAL Id: hal-01384152 \\ https://hal.science/hal-01384152
}

Submitted on 19 Oct 2016

HAL is a multi-disciplinary open access archive for the deposit and dissemination of scientific research documents, whether they are published or not. The documents may come from teaching and research institutions in France or abroad, or from public or private research centers.
L'archive ouverte pluridisciplinaire HAL, est destinée au dépôt et à la diffusion de documents scientifiques de niveau recherche, publiés ou non, émanant des établissements d'enseignement et de recherche français ou étrangers, des laboratoires publics ou privés. 


COMMUNICATION A CONGRES
Three-Dimensional Reconstructions
in Microscopy From
Two-Dimensional Interferograms
Using Sparsity-Inducing
Regularization

A. Bonnefois, L. Mugnier, A. Houillot,
G. Druart, L. Blanco
Imaging and Applied Optics,
Optics and Photonics Congress
HEIDELBERG, ALLEMAGNE
25-28 juillet 2016
TP 2016-652

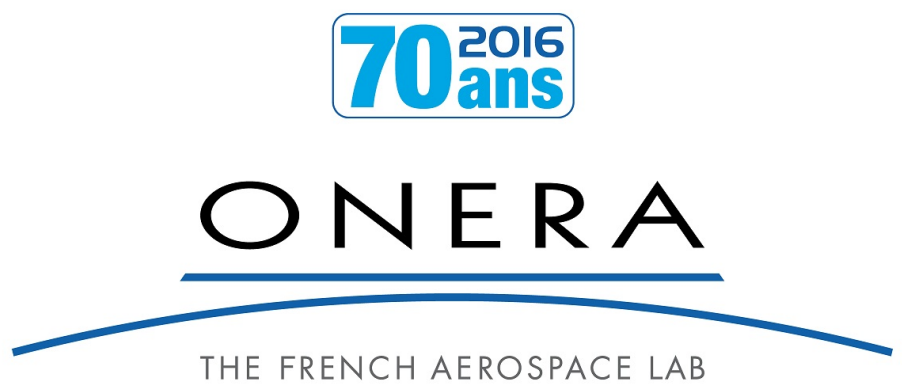





\title{
Three-Dimensional Reconstructions in Microscopy From Two-Dimensional Interferograms Using Sparsity-Inducing Regularization
}

\author{
A. Montmerle Bonnefois, L. M. Mugnier, A. Houillot, G. Druart, L. Blanco \\ ONERA, the French Aerospace Lab, Chemin de la Hunire, 91123 Palaiseau, FRANCE \\ aurelie.bonnefois@onera.fr
}

\begin{abstract}
Several applications, in the context of microscopy, need to recover the 3D information of an object consisting of a set of incoherent point-sources. We develop and validate a method that efficiently reconstructs such sparse objects in $3 \mathrm{D}$ from a $2 \mathrm{D}$ image, obtained with an incoherent-light technique such as Conoscopic Holography or Continuously Self-Imaging Grating (CSIG) imaging, which allow an efficient coding of the 3D position of a source on a $2 \mathrm{D}$ interferogram.
\end{abstract}

OCIS codes: $000.0000,999.9999$.

\section{Introduction}

The precise recovery of the 3D position of a set of point-sources is an important problem in fluorescence microscopy. Doing this task efficiently requires that it be done from a single 2D acquisition, which in turns requires a PSF that performs an efficient coding of the 3D position of any considered point-source. Several techniques have this feature, among which: PSF engineering [1], incoherent-light holography such as Conoscopic Holography (CS) [2] or FINCH [3], and Continuously Self-Imaging Grating (CSIG) imaging [4,5]. In this communication we develop and validate a reconstruction method that efficiently recovers a 3D sparse object from 2D data recorded with any of these techniques.

\section{Imaging model and reconstruction method}

We assume that the imaging device and the 3D field are chosen so that imaging is isoplanatic. The recorded 2D data is then one slice of the 3D convolution of the unknown object $o$ with the 3D PSF $h$. Discretizing the object and the PSF leads to:

$$
i(j, k)=\sum_{m}\left[h\left(\cdot, \cdot,-z_{m}\right) \star o\left(\cdot, \cdot, z_{m}\right)\right](j, k)+n(j, k)
$$

where $h$ is the 3D discrete PSF, $o$ is the 3D discrete object, $\star$ denotes 2D discrete convolution, and $n$ is the unavoidable noise (often predominantly photon noise, and possibly detector noise). For conciseness we define the PSF and the object of the m-th plane as $h_{m}=h\left(\cdot, \cdot,-z_{m}\right)$ and $o_{m}=o\left(\cdot, \cdot, z_{m}\right)$.

Following the Bayesian Maximum A Posteriori (MAP) approach, we define the solution $\hat{o}$ of the reconstruction problem as the minimizer of a metric $J(o)$ composed of the negative log-likelihood of the data plus a regularization term. Approximating the mixture of photonic and detector noises as non-stationary white Gaussian [6] yields:

$$
J(o)=\sum_{j, k} w(j, k) \cdot\left|i(j, k)-\sum_{m}\left[h_{m} \star o_{m}\right](j, k)\right|^{2}+R(o),
$$

where the weights $w(j, k)$ are the inverse of the noise variance at each pixel, and $R$ denotes the chosen regularization functional. An appropriate prior to induce sparsity in the reconstruction is the $\ell_{1}$ norm, also known as lasso regularization, or a differentiable approximation of the latter such as the one used in [7].

\section{Validation by simulations}

The efficiency of this method strongly depends whether the 3D PSF $h$ efficiently codes the distance between planes or not. The more decorrelated the PSF planes are between each other, the more precise the localisation of the point sources. 


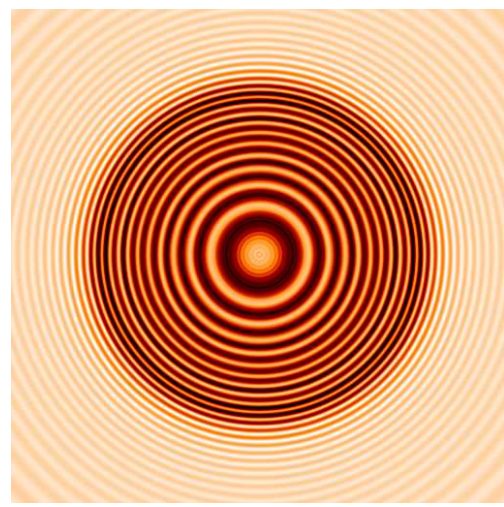

Fig. 1. Example of a defocused plane of the 3D PSF with the three imaging techniques we studied.

Left : conoscopic holography; middle : classic imaging with a circular pupil; right : CSIG
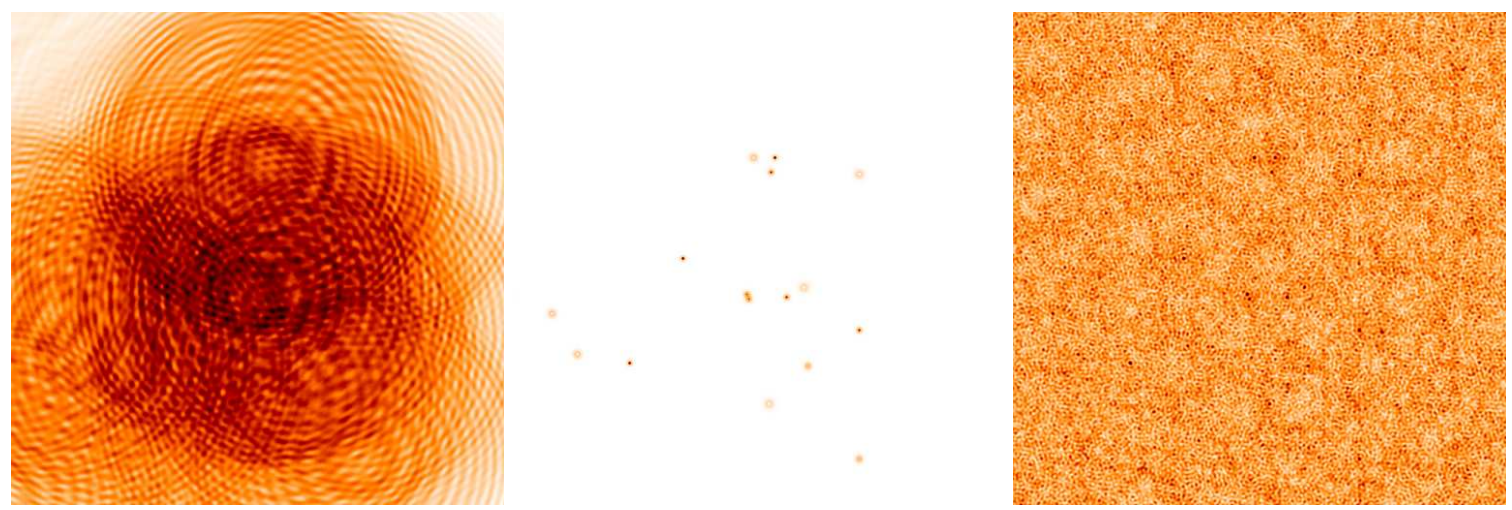

Fig. 2. Example of images of a 3D object made of several point sources randomly put in a 3Dspace, with the three imaging techniques we studied. Left : conoscopic holography; middle : classic imaging with a circular pupil; right : CSIG

Thus, we chose to test this method with two imaging techniques possessing interesting PSFs properties : holographic conoscopy [2] and CSIG imaging [4,5]. We then compared the result obtained with these two methods with traditional imaging through a circular pupil plane.

Examples of the three kinds of PSFs we studied in parallel can be shown in Figure 1. We can see that traditional imaging concentrates the energy, while CSIG and conoscopy spread it in the field. However, the diameter of the thin rings of the planes of the conoscopic 3D PSF, as well as the CSIG caracteristic diffractive pattern scale, change quickly and abruptly when they're defocused. This helps the deconvolution a lot, compared to traditional imaging, for which the PSF of defocused planes is blurry and varies slowly.

With these PSFs, we can easily simulate the images of 3D objects consisting of several point sources spread randomly in the three-dimensional space, thanks to Eq 1. An example of these images with the three techniques is shown in figure 2.

We can see on these images that without the reconstruction method, the conoscopic holography and the CSIG interferograms are difficult to interpret intuitively, while the traditional imaging technique provides images we're used to, in contexts such as microscopy for instance.

However, we've shown, both theoretically and experimentally, that the longitudinal resolution could be improved at least five times while using conoscopy and CSIG, compared to tradition imaging, as can be seen in Figure 3.

Recent results show theoretical longitudinal resolutions better than $500 \mathrm{~nm}$ obtained with this reconstruction method and conoscopy, for a microscope whose transverse resolution is $1.5 \mu \mathrm{m}$. 


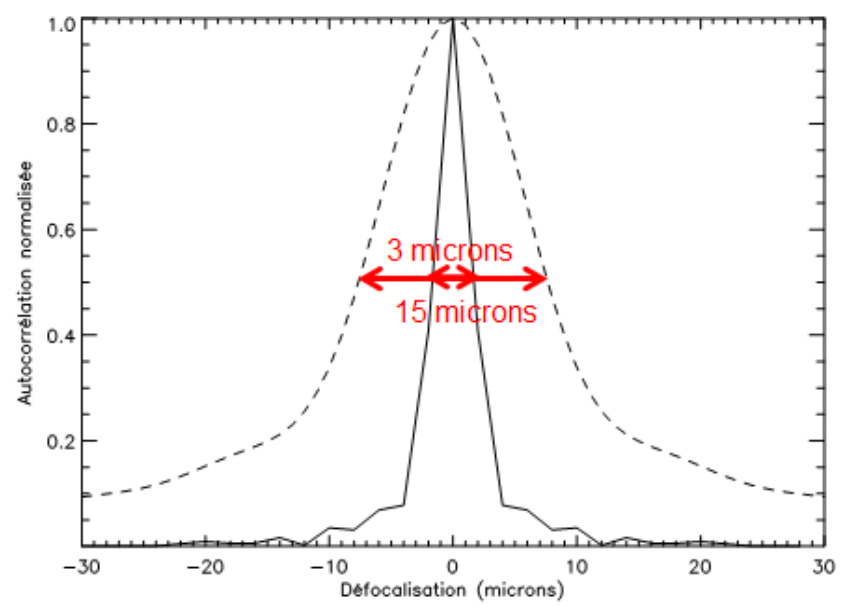

Fig. 3. Improvement of the longitudinal resolution with conoscopic holography (plain line) compared to traditional imaging (doted line) in the exact same configuration], after 3D reconstruction.

\section{Outlook}

Numerical Experiments are ongoing. A thorough performance study of this reconstruction method is currently being made, used alongside these three promising imaging techniques, in the realistic context of fluorescence microscopy.

Next, experimental validation will be studied. We already have obtained sub-micron experimental longitudinal resolution with a CSIG device used alongside fluorescence microscopy of point sources balls in a transparent gel. Similar experiments are to be done in the forseeable future. The dynamical range and the resolutions of the three imaging techniques used with this reconstruction method will then be compared on experimental data.

\section{References}

1. Y. Shechtman, J. S. Sahl, A. S. Backer, W. E. Moerner, Optimal Point Spread Function Design for 3D Imaging, Phys. Rev. Lett., 113, 133902 (2014).

2. L. Mugnier, Conoscopic holography: towards three-dimensional reconstructions of opaque objects, Applied optics, 34(8), p. 1363-1371. (1995).

3. J. Rosen and G. Brooker, Non-scanning motionless fluorescence three-dimensional holographic microscopy, Nature Photonics, 2(190) (2008).

4. G. Druart, R. Horisaki, M. Piponnier, N. Gurineau, J. Primot, L. Mugnier, A 20th way to do 3-D imaging using a Continuously Self Imaging Grating (CSIG), Proceedings of COSI, OSA (2015).

5. M. Piponnier, G. Druart, R. Horisaki, N. Gurineau, J. Primot, L. Mugnier, F. Goudail, Three-dimensional imaging using continuously self-imaging gratings., Optics letters 38(20), p. 4058-4061. (2013).

6. L. Mugnier, T. Fusco, and J.-M. Conan, MISTRAL: a myopic edge-preserving image restoration method, with application to astronomical adaptive-optics-corrected long-exposure images, J. Opt. Soc. Am. A, 21(10), 1841-1854,(2004).

7. S. Meimon, L. Mugnier, and G. Le Besnerais, Self-calibration approach for optical long-baseline interferometry imaging, J. Opt. Soc. Am. A, 26(1), 108-120, (2009). 
Office National d'Études et de Recherches Aérospatiales Chemin de la Hunière et des Joncherettes - BP 80100 91123 PALAISEAU Cedex

Tél. : +33180386060-Fax : +33180386510 http://www.onera.fr 